\title{
The Resistivity of Human Brain Tumours In Vivo
}

\author{
J. LATIKKA and H. ESKola \\ Faculty of Biomedical Sciences and Engineering, Tampere University of Technology, Tampere, Finland \\ (Received 3 July 2018; accepted 13 December 2018; published online 4 January 2019)
}

Associate Editor Karol Miller oversaw the review of this article.

\begin{abstract}
The histological structure of tumour tissues differs from healthy brain tissues. It can therefore be assumed that there are differences also in the electrical characteristics of these tissues. The electrical characteristics of the tissues define how electric current is distributed within volume conductors, such as the human body or head. Incorrect values affect, for example, the accuracy of impedance tomography or EEG source localisation. However, no controlled experimental data for human in vivo brain tumour resistivity values have been reported thus far. We have developed a controlled method for detecting the electrical resistivities of living brain tissue and investigated different types of brain tumours. The measurements were taken during brain surgeries conducted to remove the tumours. For analysis purposes, the tumours were divided into the following categories: meningiomas, low-grade gliomas, high-grade gliomas (glioblastomas) and other tumours or lesions. The averages of the measured resistivity values were $530 \Omega$-cm for meningiomas, $160 \Omega$-cm for low-grade gliomas, and $498 \Omega$ $\mathrm{cm}$ for high-grade gliomas. The differences in high- and lowgrade glioma values and meningioma and low-grade glioma values were statistically highly significant. The tumour values were also compared to surrounding healthy brain tissues, and the difference ranged from 40 to $330 \%$. The results suggest that certain tumour types have different electronic properties and that the resistivity values could be used to distinguish tumour tissue from surrounding healthy tissue and to identify and classify certain brain tumour types.
\end{abstract}

Keywords-Tissue electrical properties, Conductivity, Modelling.

\section{INTRODUCTION}

The electrical properties of brain tissue are interesting for several purposes. For instance, volume conductor models for electroencephalogram (EEG) source analysis are based on the geometry and algorithms, but also on the conductivity data, of all tissues

Address correspondence to J. Latikka, Faculty of Biomedical Sciences and Engineering, Tampere University of Technology, Tampere, Finland. Electronic mail: latikka@student.tut.fi in the models. Incorrect values in the models may cause source localisation errors. ${ }^{13}$ Electrical properties are also necessary in estimating the specific absorption rate of cell phone radiation to the human brain or the absorption of any other electromagnetic radiation, such as short-range energy transfer, in calculating fields in transcranial magnetic stimulation (TMS) ${ }^{23}$ or in transcranial direct-current stimulation (tDCS). ${ }^{16}$ Further, the values are beneficial in planning the treatment of tumours with radiofrequency-induced hyperthermia ${ }^{15}$ or planning transcranial direct-current stimulation treatment. ${ }^{6}$ Several researchers have expressed the need for accurate tissue and tumour values. $^{1,29,30}$

The difference in the resistivity values between different intracranial tissues has been successfully applied in neuronavigation, where impedance measurements have been used to aid in localisation during stereotactic surgeries. ${ }^{7,17}$ In these procedures, the tissue boundaries have been detected by relative impedance changes, ${ }^{4}$ but the absolute values have not been of interest. Detection of tissue boundaries has also been used in the accurate placement of deep-brain-stimulation electrodes into specific areas of the brain in the treatment of epilepsy and Parkinson's disease. ${ }^{8,19}$

The shape and size of brain lesions can be quite easily obtained by modern medical imaging methods, such as magnetic resonance imaging (MRI). Brain lesions can have various shapes but data on their electrical conductivities are scarce. They may have a much higher conductivity than brain tissue (e.g., oedemas) or a much lower one (calcifications). Some brain tumours are quite homogeneous and some consist of various kinds of tissues, especially when necrosis has already developed. This means that it is impossible to give any general model for brain tumour conductivity. It is nonetheless expected that the conductivity follows the blood and other liquid contents of the tumour tissue. 
There are previous studies that indicate that the tumour tissue does in fact have different electrical characteristics compared to a surrounding healthy tissue. Literature knows examples of measurements taken from animal tissue samples, ${ }^{2}$ from animals in vivo, ${ }^{25}$ from human brain tissue samples ${ }^{1}$ and from other human tissues. ${ }^{27}$ In addition, in vivo differences from healthy human brain and tumour tissues have been recorded in stereotaxic operations. ${ }^{4,21}$ However, as mentioned before, the interest in stereotactic surgery lies in detecting tissue boundaries by differences in the measured values. The results of these measurements do not take into account electrode areas, placements and the surrounding body and therefore most likely differ from absolute resistivity values.

Although there is information on human tumour conductivities measured in vitro and in vivo, it is important to have calibrated in vivo measurements for accurate modelling and simulations. The presence of a lesion with a different electrical conductivity than the surrounding tissues may alter EEG and magnetoencephalography (MEG) results and the source localisation procedure may fail if the lesion is not properly included in the volume conductor model. ${ }^{28,30}$ Given the variation in reported values in literature, using inaccurate tissue values may cause a large localisation error. ${ }^{29}$ In addition, Haemmerich et al. ${ }^{11}$ demonstrated the importance of in vivo measurements and showed that there is a significant change in tissue resistivity after removal of the organ and that accurate results require in vivo measurements with intact circulation. Robinson et al. ${ }^{22}$ reported that there is an increase of $2700 \Omega$ baseline resistance in the brain perfused with $10 \%$ formalin (formaldehyde) compared to the living brains of macaca mulatta. However, the records were often of better quality than those obtained in the living brain, and the authors presumed this was due to the removal of minor impedance noise related to vascular factors.

One important aspect in conductivity measurements is the measurement frequency. The relative permittivity $(\varepsilon)$ and conductivity $(\sigma)$ of the tissues depend strongly on frequency. This frequency dependence (dispersion) arises from several mechanisms. For a typical soft tissue, different mechanisms dominate at different frequency ranges. ${ }^{9}$ Electrical properties change with frequency in three distinct steps, known as alpha $(\alpha)$, beta $(\beta)$ and gamma $(\gamma)$ dispersions. ${ }^{24}$ The low-frequency $\alpha$ dispersion is associated with ionic diffusion processes at the site of the cellular membrane. The $\beta$ dispersion, in the hundreds of kilohertz region, is mainly due to the polarisation of cellular membranes, which act as barriers to the flow of ions between the intra- and extracellular media. Other contributions to the $\beta$ dispersion come from the polarisation of protein and other organic macromolecules. The $\gamma$ dispersion, in the gigahertz region, is due to the polarisation of water molecules. ${ }^{10}$ Since all bioelectrical sources produce frequencies below $1 \mathrm{kHz}$ only, a low measurement frequency is necessary especially for source localisation modelling purposes.

In this study, we have taken calibrated measurements using a measurement frequency well below the $\beta$ dispersion area from living human brain tissue to find out the electrical resistivity of human brain tumours in vivo. The measurements were taken using monopolar measurement technique from 20 patients with brain lesions. Our interest was in determining absolute values for tumour tissue resistivity, because the absolute values could aid in constructing more accurate brain and head models. The recorded in vivo resistivity values showed differences between tumour types and surrounding brain tissues.

\section{MATERIALS AND METHODS}

The measurements were taken from living tissues during surgeries. The surgeries were normal operations that aimed to remove lesions, except for the tissue resistivity measurements. The measurements were obtained before the removal procedures with intact circulation in the target to avoid changes in resistivity due to a changed blood volume within the tumour. The measurements did not disturb the progress of the surgery and did not cause any additional damage to the patients. When the surgical channel went through the grey and white matters of the brain, or ventricles containing cerebrospinal fluid (CSF), measurements were also taken from these tissues. The measurements were approved by the ethical council of Tampere University Hospital.

The tumour resistivity values were measured with FL Fischer Neuro N50. The Neuro N50 is designed to be used in minimally invasive pain treatment and neurosurgery, and it has been approved for operation room environment use. The system uses $50 \mathrm{kHz} 2 \mu \mathrm{A}$ sinusoidal current for the impedance measurement. It has several options for electrodes. In this study we used the TCU 301 electrode inside a guide cannula with an exposed $2 \mathrm{~mm}$ tip as the small active electrode for the monopolar measurements. As reference electrodes, we used disposable electrodes with an area of $130 \mathrm{~cm}^{2}$. The reference electrodes were usually placed on the hip or thigh area of the patient. If, for some reason, the reference electrode had lost contact, the Neuro N50 would not have given any measurement readings. The measurement setup was the same as in Latikka et al. ${ }^{18}$

The measurement range of Neuro N50 ranges from 0 to $2040 \Omega$ with a $1 / 10 \Omega$ resolution. The linearity of 
the device and the accuracy of the impedance measurements were tested before the tissue measurements. First, ohmmeter and resistor networks were used, then commercial conductivity cell with liquids of different resistivities. These measurements were made within the measurement range of the equipment. The measurement range of Neuro N50 proved sufficient for intracranial tissue impedance measurements since all measured impedance values were less than $2000 \Omega$.

The resistivity of the saline wash, which was used for rinsing during the surgeries and as reference liquid for resistivity calculations, was also measured in a laboratory. Measurements from the saline wash were taken at 25-44 degrees Celsius, exceeding the range of the temperatures of the used saline wash during the surgeries. As saline is an ionic liquid, its resistivity is temperature-dependent, and therefore the temperature needs to be considered when calculating the final results. Both the resistivity and the temperature of the saline wash were measured during the surgeries and compared to the value measured in the laboratory to account for the effect of the human body on the resistivity measurements and to control possible equipment drifting. The possible drifting of the equipment during the surgeries was controlled by taking measurements from reference liquid when it was used for rinsing. The rinsing usually took place several times during the operations.

With monopolar measurement techniques, the measurement result is heavily weighted to the immediate surroundings of the small active electrode (the needle tip in this study). When the reference liquid is measured in vivo during surgery, and when we have prior knowledge of the resistivity of the reference liquid, we can calculate the baseline introduced by the human body to the measured values. Knowing this individual baseline value, we can estimate the resistivities of measured intracranial tissues for each patient.

The measurements were made on 20 patients aged 4-87 years. One patient had an arteriovenous (AV) malformation and another patient had an old haemorrhage. In one case it was not possible to reliably record the tumour value. Hence, only measurements from 17 patients were included in the tumour value analysis. The number of measurements and the number of different tissues measured per each patient depended on the tumour or lesion location and the surgical path. Figure 1 shows some examples of the measurement locations.

With diffusing tumours, particular care was taken to ensure that the measurements were obtained from the tumour and not from the diffused area. Also, with measurements from white or grey matter, the aim was

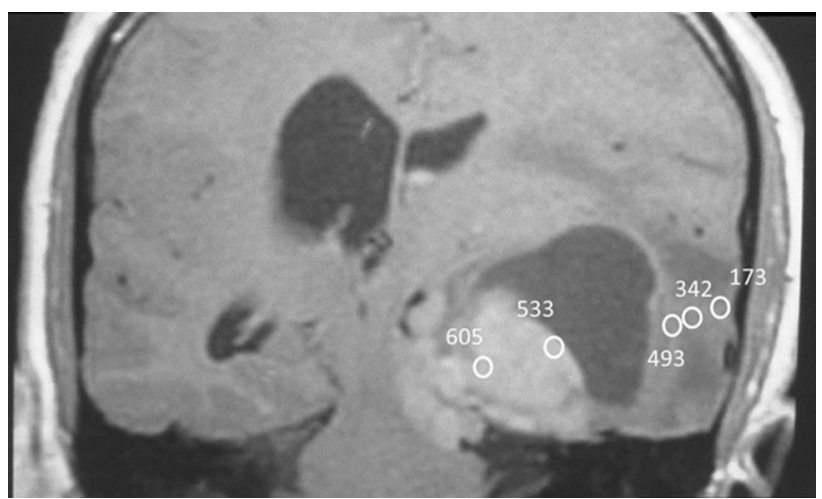

FIGURE 1. T1 (TR: 600, TE:11) MR image of patient GH1. The measurement locations are indicated by the white circles with their respective resistivity values.

to measure healthy tissue, if possible. For example, oedematous white matter has been reported to have generally a lower value when compared to non-oedematous white matter. ${ }^{5}$ This is most likely also true for grey matter.

For analysis purposes, the tumours were divided into the following categories: meningiomas (M), lowgrade gliomas (GL), high-grade gliomas (GH) (glioblastomas) and other tumours or lesions. Meningiomas are solid tumours and have distinct boundaries whereas gliomas are cystic and may diffuse into surrounding tissue, blurring the boundaries. The other tumours or lesions measured were one pineal tumour, one haematoma and one AV malformation.

The number of measurements per patient and per tissue depended on the surgery and ranged from 1 to 15 for high-grade gliomas, from 3 to 17 for low-grade gliomas, from 1 to 11 for meningiomas, from 1 to 6 for grey matter, from 1 to 12 for white matter, 3 for AV malformation, 5 for pineal tumour and 1 for old haematoma. The number of measurements for each patient is shown in Table 1.

For analysis purposes, the tumour values were considered independent, since no patient had more than one tumour type. The average resistivity was calculated for each tumour and the averages were compared between tumour types. Preliminary f-tests for the equality of variances were conducted for each tissue pair to investigate whether the variances of the two tumours were significantly different. Based on the result, a two-sample t-test was performed that assumed or did not assume equal variances to investigate if different tumour types could be identified by resistivity measurements. Only meningiomas, high-grade gliomas and low-grade gliomas were included in these analyses, since other lesion types had only one set of measurements. 
TABLE 1. Patient code, age, brain tumour type and number of measurements taken from each measured tissue type: white matter, grey matter and tumour.

\begin{tabular}{|c|c|c|c|c|c|}
\hline \multirow[b]{2}{*}{ Patient code } & \multirow[b]{2}{*}{ Age } & \multirow[b]{2}{*}{ Brain tumor type } & \multicolumn{3}{|c|}{ Number of measurements } \\
\hline & & & White matter & Grey matter & Tumour \\
\hline $\mathrm{GH} 1$ & 32 & High-grade glioma & & & 2 \\
\hline GH2 & 64 & High-grade glioma & 1 & & 6 \\
\hline $\mathrm{GH} 3$ & 48 & High-grade glioma & 12 & 6 & 15 \\
\hline $\mathrm{GH} 4$ & 63 & High-grade glioma & & 3 & 1 \\
\hline GH5 & 73 & High-grade glioma & 3 & 2 & 3 \\
\hline GL1 & 70 & Low-grade glioma & 2 & 6 & 8 \\
\hline GL2 & 33 & Low-grade glioma & 4 & 3 & 5 \\
\hline GL3 & 4 & Low-grade glioma & & 4 & 3 \\
\hline GL4 & 52 & Low-grade glioma & & & 17 \\
\hline M1 & 61 & Meningioma & 2 & 4 & \\
\hline M2 & 87 & Meningioma & & 2 & 3 \\
\hline M3 & 69 & Meningioma & & & 3 \\
\hline M4 & 54 & Meningioma & & 1 & 1 \\
\hline M5 & 49 & Meningioma & 4 & 4 & 3 \\
\hline M6 & 77 & Meningioma & & & 11 \\
\hline M7 & 54 & Meningioma & & 1 & 3 \\
\hline M8 & 66 & Meningioma & & & 3 \\
\hline $\mathrm{H} 1$ & 67 & Haematoma & & & 1 \\
\hline P1 & 50 & Pineal tumour & & & 5 \\
\hline AV1 & 61 & AV malformation & & 3 & 3 \\
\hline
\end{tabular}

\section{RESULTS}

Table 2 shows the average of measurement results in $\Omega$-cm for each patient. The measured values for the brain tumours and lesions are given with grey and white matter values, if available. The white or grey matter measurements were taken only if the surgical path passed through healthy white or grey matter. There were no measurements taken outside the surgical path to avoid any additional damage to the patient. In some cases, measurements were taken also during the removal procedure, but these values were not included in the reported values or statistical analysis. The average values, with minimum and maximum values of the measured tumours, are presented in Fig. 2.

Different tumour types were compared to see whether the tumour types could be identified by resistivity measurements. Only meningiomas, high-grade gliomas and low-grade gliomas were included in this analysis, since other lesion types had only one set of measurements, as mentioned above.

The preliminary tests ( $f$-tests) for the equality of variances for tumour tissues showed that each investigated tumour pair had equal variances. Based on the f-tests, two-sample t-tests were performed that assumed equal variances.

The mean value for high-grade gliomas $(M=498$, $\mathrm{SD}=109, N=5$ ) was significantly higher than for low-grade gliomas $(M=160, \mathrm{SD}=60, N=4)$ using the two-sample t-test for equal variances, $t(7)=5.49$, $p \leq 0.001$. Also, the mean value for meningiomas $(M=530, \mathrm{SD}=178, N=7)$ was significantly higher than for low-grade gliomas using the two-sample t-test for equal variances, $t(9)=-3.95, p \leq 0.004$. The mean values for meningiomas and high-grade gliomas showed no statistical differences using the two-sample t-test for equal variances, $t(10)=-0.36, p \leq 0.73$. Grey matter had a mean value of $306 \Omega-\mathrm{cm}$ $(\mathrm{SD}=133, N=8)$ and white matter a mean value of $372 \Omega-\mathrm{cm}(\mathrm{SD}=93, N=12)$.

\section{DISCUSSION}

The variance in the measured data is quite high. However, this is in line with previous studies $1,12,14,26$ and most probably caused by biological variation. For example, in Haemmerich et al., ${ }^{12}$ the tumour tissue showed a much higher variance in the data acquired from tumour tissue compared to normal tissue. They explain the higher variability by the much higher heterogeneity of tumour cells. Other variables, such as tumour size, age, etc., might also influence the conductivity.

Our results show that there are differences in resistivity between different tumour types and that it may be possible to separate high-grade gliomas from lowgrade gliomas and meningiomas from low-grade gliomas based on their different electrical properties. Some previously recorded impedance values for gliomas can 


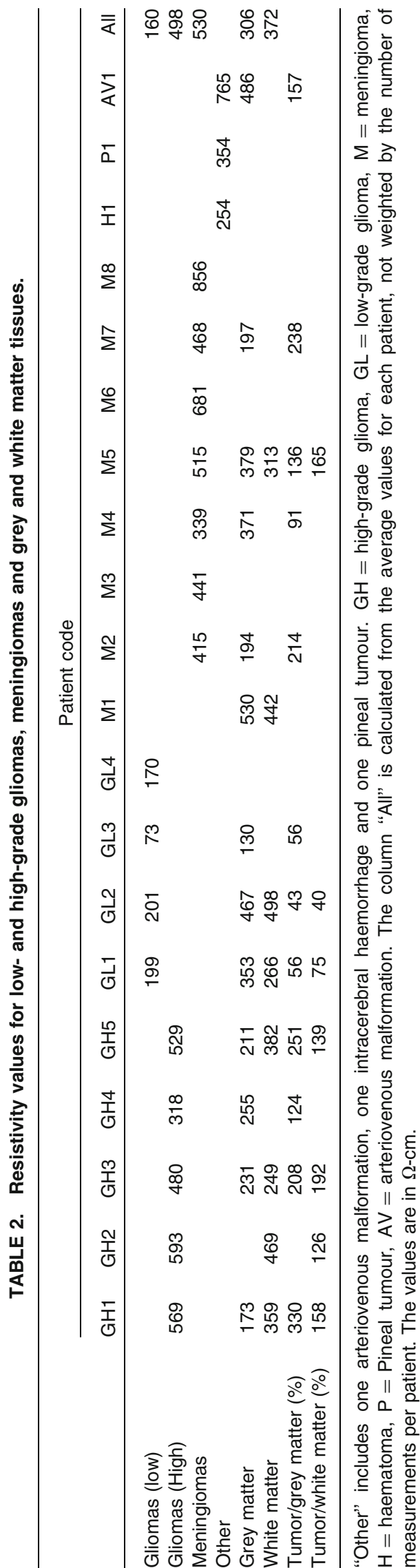

be found in literature. ${ }^{21}$ These results are in line with ours in the sense that generally lower impedance values were recorded with low-grade gliomas (grades 1 and 2) and higher values with higher-grade gliomas (grades 3 and 4). The average readings reported in Rajshekhar ${ }^{21}$ were $443 \Omega$ for lower-grade gliomas and $675 \Omega$ for higher-grade gliomas, and in the results of this study $160 \Omega$-cm for lower-grade gliomas and $498 \Omega$-cm for higher-grade gliomas.

Our approach and our interest in tumours are different than in earlier studies. Most earlier studies were aimed at continuous impedance monitoring during CT-guided stereotactic procedures, where the benefit arises from the detection of tissue boundaries, indicating the arrival of the probe in the target tissue or in easily recognisable structures in the surgical path, such as CSF-filled ventricles. Verifying tissue boundaries during stereotactic surgery requires only a sufficient change in impedance reading to give enough information to detect the tissue boundaries. When detecting tissue boundaries, artefacts caused by the reference location, etc., can be neglected as long as the reference electrode stays in contact. Therefore, the absolute values are not of interest and no measures have been made to calculate the absolute values after the operation.

Our interest was in determining absolute values for tumour tissue resistivity, which required calibration measurements before and during surgery as well as calculations after the measured values. In addition to the value of the measured tissue, electrode areas of the measurement electrode and the ground electrode, as well as the current path from the measurement electrode to the ground electrode, contribute to the reading. All these must be considered if absolute values are to be known. The clear baseline difference in the results compared to Rajshekhar ${ }^{21}$ may well be due to these factors.

To demonstrate the need for calibrated measurements, we can look at a very simple model that includes only two parallel resistors, R1 and R2. Let the shape and size of the resistors be so that with resistivities $160 \Omega$-cm (resistivity of low-grade gliomas in this study) and $372 \Omega$-cm (resistivity of white matter in this study) the resistance of R1 becomes 160 and R2 $372 \Omega$. When the potential difference $(U)$ is applied to this network, the current (I) will flow according to Ohm's law $I=U / R$, where the $R$ is the total resistance of the network. The current will be divided according to Kirchhoff's Current Laws so that $I=I 1+I 2$, where $I 1$ is the current flowing through R 1 and $I 2$ the current flowing through $\mathrm{R} 2$. The $U$ is the same for both resistors and the proportion of $I 1$ and $I 2$ of $I$ can be calculated using the resistor values: for $I 1=(\mathrm{R} 2 /$ $(\mathrm{R} 1+\mathrm{R} 2))$ and for $I 2=(\mathrm{R} 1 /(\mathrm{R} 1+\mathrm{R} 2))$. In our 


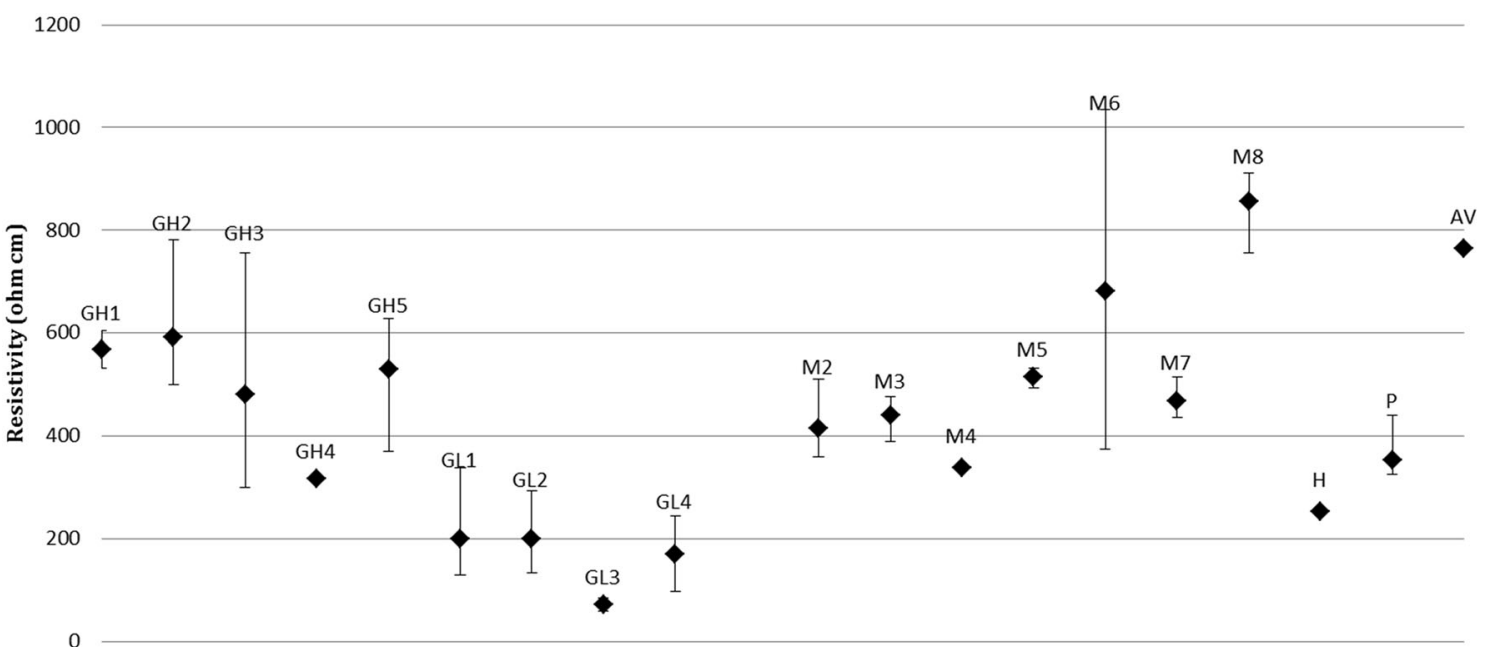

FIGURE 2. Average, minimum and maximum tumour resistivity values for each patient. GH high-grade glioma, GL low-grade glioma, $M$ meningioma, $H$ haematoma, $P$ pineal tumour, $A V$ arteriovenous malformation.

extremely simple example, approximately $70 \%$ of the current flows though R1 and 30\% through R2.

Now, if we introduce baseline resistance to the resistors in our model, the values of $\mathrm{R} 1$ and $\mathrm{R} 2$ will both change. As an example, an increase of $2700 \Omega$ baseline resistance in the brain perfused with $10 \%$ formalin (formaldehyde) compared to the living brains of macaca mulatta has been reported. ${ }^{22}$ If we add this baseline to our resistors, they will become 2860 and $3072 \Omega$. The difference is still the same, $212 \Omega$, but the ratio is not. When we re-calculate the current distribution between the resistors, we see that approximately $52 \%$ of the current flows through R1 and $48 \%$ through $\mathrm{R} 2$. Thus, the presence of the baseline leads to a more homogenous model and affects the simulation results.

In all our measurement cases, we could identify healthy tissue from tumour tissues by resistivity measurements. In Smith et al. ${ }^{26}$ it was found that the differences in the conductivity of tumour vs. normal tissue are most pronounced below $100 \mathrm{kHz}$. Our measurement frequency of $50 \mathrm{kHz}$ is below this and below the above-mentioned $\beta$ dispersion area. In Peloso et $a l .{ }^{20}$ the researchers did not observe significant differences in the measured dielectric properties of normal and neoplastic rat muscle tissues. It was presumed that this was due to the high water content of both types of tissues. This is most likely the case, since a higher measurement frequency with a range from $1 \mathrm{MHz}$ to $1 \mathrm{GHz}$ was used, where the $\beta$ and $\gamma$ dispersions become the most prominent. In addition to capacitive shorting-out of membrane resistances and rotational relaxations of biomacromolecules, the relaxation of free water in the tissues contributes to the reading. As mentioned, a low measurement frequency is especially necessary for modelling purposes, since all bioelectrical sources produce frequencies below $1 \mathrm{kHz}$ only.

In this study, in some cases the resistivity of a given tumour increased during surgery, but this might have been mainly due to the cut blood circulation to the tumour. For example, cutting the circulation to the tumour during the surgery of patient M2 increased the resistivity of the tumour. The average of the resistivity values from tumour was $415 \Omega-\mathrm{cm}$ when circulation was intact and $614 \Omega-\mathrm{cm}$ after the circulation was cut. Thus, to obtain correct conductivity values, the measurements should be made in vivo and at the beginning of the surgery. The results of Haemmerich et al. ${ }^{11}$ support this. The measurement taken after the circulation was cut were not used in the results section of this study and therefore do not affect the reported values in Table 2.

It has been demonstrated with mathematical models that incorrect lesion conductivity assignment leads to errors in source localisation with highly conductive lesions and if the source is close to a lesion. ${ }^{28,30}$ On the other hand, Broedbeck et al. ${ }^{3}$ states that their results suggest that electromagnetic source imaging can identify the epileptogenic focus with excellent accuracy in most patients despite volume-relevant brain lesions that most likely change conductivity properties within the skull. They used a three-shell model for the head, and the precision of the method was evaluated with respect to the surgical resection and surgical outcome. Vatta et $a l .{ }^{30}$ used a simple four-sphere model.

When tumours grow, they may stimulate oedematous or inflammatory effects. At a later stage, the tumours will also become necrotic, which will change the conductivity value within the tumour, as this will increase the amount of liquid within the tissue, thus 
increasing the conductivity. In the measurements of this study, the necrotic areas were easily recognised during surgery, and measurements from these locations were not included in the results presented in Table 2. In the case of patient GH2, the measurements taken from the necrotic area showed $490 \Omega$-cm resistivity, which is approximately $100 \Omega$-cm lower than that of the surrounding tumour tissue. The effects of oedema and necrosis have been discussed in earlier studies. ${ }^{5,25}$ However, they stated that further experimentation is needed to determine the microscopic cause of the observed changes.

The method used in this paper has its disadvantages, for example, it does not account for the possible anisotropy of the tumour tissues. The challenging operating theatre conditions, including taking measurements during surgical procedures, brought naturally restrictive elements to the study. Grey matter and white matter measurements are very sensitive in terms of measurement locations, and they were dependent on the measurement instrument location information provided by the surgeon and not verified histologically. Therefore, this may have been an additional cause of variation.

In this study, the tumour resistivity was found to range from 40 to $330 \%$ of the surrounding brain tissue values. It is most likely that this difference is high enough to affect the simulations based on the conductivity models for EEG studies, radiofrequency exposure or impedance tomography, especially if the region of interest is near a tumour. More conductivity measurements (either invasive or non-invasive) calibrated to produce actual values should be performed to confirm these conclusions. The measurements should be performed with low enough frequencies and from living tissues. With additional data, the values could be even used for diagnostic purposes.

We conclude that both realistic shapes and conductivities of tumours are necessary for accurate conductivity modelling of the head. The real effect of the tumours can be evaluated only by applying the conductivity values to each specific case. We plan to investigate whether the tumours will affect the results of source location studies when using the values reported here in comparison to the values used in earlier studies.

Based on our statistical analysis presented in this study, we can conclude that there are differences in conductivities between different tumour types and that it may be possible to separate high-grade gliomas from low-grade gliomas and meningiomas from low-grade gliomas based on their different electrical properties. However, we emphasise that the final diagnosis should be made through histological assessment only.

We also compared the tumour values to surrounding healthy brain tissues, and the difference ranged from 40 to $330 \%$. The results suggest that certain tumour types have different electrical properties and that the resistivity values could be used to distinguish tumour tissues from surrounding healthy tissues and to identify and classify certain brain tumour types.

\section{CONFLICT OF INTEREST}

No benefits in any form have been or will be received from a commercial party related directly or indirectly to the subject of this manuscript.

\section{OPEN ACCESS}

This article is distributed under the terms of the Creative Commons Attribution 4.0 International License (http://creativecommons.org/licenses/by/4.0/), which permits unrestricted use, distribution, and reproduction in any medium, provided you give appropriate credit to the original author(s) and the source, provide a link to the Creative Commons license, and indicate if changes were made.

\section{REFERENCES}

\footnotetext{
${ }^{1}$ Akhtari, M., M. Mandelkern, D. Bui, N. Salamon, H. V. Vinters, and G. W. Mathern. Variable anisotropic brain electrical conductivities in epileptic foci. Brain Topogr. 23:292-300, 2010.

${ }^{2}$ Blad, B., and B. Baldetorp. Impedance spectra of tumour tissue in comparison with normal tissue; a possible clinical application for electrical impedance tomography. Physiol. Meas. 17:A105-A115, 1996.

${ }^{3}$ Broedbeck, V., A. M. Lascano, L. Spinelli, M. Seeck, and C. M. Michel. Accuracy of EEG source imaging of epileptic spikes in patients with large brain lesions. Clin. Neurophysiol. 120:679-685, 2009.

${ }^{4}$ Bullard, D. Intraoperative impedance monitoring during CT-guided stereotactic biopsies. Stereotact. Funct. Neurosurg. 52:1-17, 1989.

${ }^{5}$ Bullard, D., and T. Makachinas. Measurement of tissue impedance in conjunction with computed tomographyguided stereotaxic biopsies. J Neurol Neurosurg Psychiatry 50:43-51, 1987.

${ }^{6}$ Datta, A., J. M. Baker, M. Bikson, and J. Fridriksson. Individualized model predicts brain current flow during transcranial direct-current stimulation treatment in responsive stroke patient. Brain Stimul. 4:169-174, 2011.

${ }^{7}$ Dierssen, G., and E. Marg. The value of impedance measurements to aid in the localisation in stereotactic surgery. Confin. Neurol. 26:407-410, 1965.

${ }^{8}$ Eugster, L., P. Bargiotas, C. L. Bassetti, and W. M. S. Schuepbach. Deep brain stimulation and sleep-wake functions in Parkinson's disease: a systematic review.
} Parkinsonism Relat. Disord. 32:12-19, 2016. 
${ }^{9}$ Foster, K. R. Dielectric properties of tissues. In: The Biomedical Engineering Handbook, edited by J. D. Bronzino. Boca Raton: CRC Press, 1995, pp. 1385-1394.

${ }^{10}$ Gabriel, C., S. Gabriel, and E. Corthout. The dielectric properties of biological tissues: I. Literature survey. Phys. Med. Biol. 41:2231-2249, 1996.

${ }^{11}$ Haemmerich, D., O. R. Ozkan, J.-Z. Tsai, S. T. Staelin, S. Tungjitkusolmun, D. M. Mahvi, and J. G. Webster. Changes in electrical resistivity of swine liver after occlusion and post-mortem. Med. Biol. Eng. Comput. 40:29-33, 2002.

${ }^{12}$ Haemmerich, D., S. T. Staelin, J. Z. Tsai, S. Tungjitkusolmun, D. M. Mahvi, and J. G. Webster. In vivo electrical conductivity of hepatic tumours. Physiol. Meas. 24:251260, 2003.

${ }^{13}$ Haueisen, J., C. Ramon, M. Eiselt, H. Braurer, and H. Nowak. Influence of tissue resistivities on neuromagnetic fields and potentials studied with a finite element and a boundary element model of the head. IEEE Trans. Biomed. Eng. 44:727-735, 1997.

${ }^{14}$ Iacono, R., J. Carlson, S. Kuniyoshi, Y. Li, A. Mohamed, and G. Maeda. Electrophysiologic target localization in postroventral pallidotomy. Acta Neurochir. 139:433-441, 1997.

${ }^{15}$ Johansson, J. D., D. Loyd, K. Wårdell, and J. Wren. Impact of cysts during radiofrequency lesioning in deep brain tissues - a simulation and in vitro study. J. Neural Eng. 4:87-95, 2007.

${ }^{16}$ Laakso, I., S. Tanaka, M. Mikkonen, S. Koyama, N. Sadato, and A. Hirata. Electric fields of motor and frontal tDCS in a standard brain space: a computer simulation study. NeuroImage 137:140-151, 2016.

${ }^{17}$ Laitinen, L., and G. Johansson. Locating human cerebral structures by the impedance method. Confin. Neurol. 29:197-201, 1967.

${ }^{18}$ Latikka, J., T. Kuurne, and H. Eskola. Conductivity of living intracranial tissues. Phys. Med. Biol. 46:1611-1616, 2001.

${ }^{19}$ Lehtimäki, K., T. Möttönen, K. Järventausta, J. Katisko, T. Tähtinen, J. Haapasalo, T. Niskakangas, T. Kiekara, J. Öhmana, and J. Peltola. Outcome based definition of the anterior thalamic deep brain stimulation target in refractory epilepsy. Brain Stimul. 9:268-275, 2016.

${ }^{20}$ Peloso, R., D. Tuma, and R. Jain. Dielectric properties of solid tumors during normothermia and hyperthermia. IEEE Trans. Biomed. Eng. 32:725-728, 1984.
${ }^{21}$ Rajshekhar, V. Continuous impedance monitoring during CT-guided stereotactic surgery: relative value in cystic and solid lesions. Br. J. Neurosurg. 6:439-444, 1992.

${ }^{22}$ Robinson, B., J. Bryan, and H. Rosvold. Locating Brain Structures - extensions to the impedance method. Arch. Neurol. 13:477-486, 1965.

${ }^{23}$ Salinas, F. S., J. L. Lancaster, and P. T. Fox. 3D modeling of the total electric field induced by transcranial magnetic stimulation using the boundary element method. Phys. Med. Biol. 54:3631-3647, 2009.

${ }^{24}$ Schwan, H. P. Electrical of tissues and cell suspensions: mechanisms and models. Engineering in Medicine and Biology Society. Engineering advances: new opportunities for biomedical engineers. Proceedings of the 16th Annual Conference of the IEEE 1, pp. A70-A71, 1994.

${ }^{25}$ Skourou, C., J. P. Hopes, R. R. Strawbridge, and K. D. Paulsen. Feasibility studies of electrical impedance spectroscopy for early tumor detection in rats. Physiol. Meas. 25:335-346, 2004.

${ }^{26}$ Smith, S., K. Foster, and G. Wolf. Dielectric properties of VX-2 carcinoma versus normal liver tissue. IEEE Trans. Biomed. Eng. 33:522-524, 1986.

${ }^{27}$ Surowiec, A. J., S. S. Stulchy, J. R. Barr, and A. Swarup. A dielectric properties of breast carcinoma and the surrounding tissues. IEEE Trans. Biomed. Eng. 35:257-263, 1988.

${ }^{28}$ Van den Broek, S. P., F. Reinders, M. Donderwinkel, and M. J. Peters. Volume conduction effects in EEG and MEG. Electroencephalogr. Clin. Neurophysiol. 106:522-534, 1998.

${ }^{29}$ Van Uiert, R., C. Johnson, and L. Zhukow. Influence of head tissue conductivity in forward and inverse magnetoencephalographic simulations using realistic head models. IEEE Trans. Biomed. Eng. 51:2129-2137, 2004.

${ }^{30}$ Vatta, F., P. Bruno, and P. Inchingolo. Improving Lesion conductivity estimate by means of EEG source localization sensitivity to model parameter. J. Clin. Neurophysiol. 19:115, 2002.

Publisher's Note Springer Nature remains neutral with regard to jurisdictional claims in published maps and institutional affiliations. 